\title{
LncRNA-H19 Induces Retinal Müller Cell Apoptosis via MiR-29b/ FOXO4 Axis in Diabetic Retinopathy
}

\author{
Qiu-li Zhang, ${ }^{\mathrm{a}, *, \#, \text { Wei Wang, }}{ }^{\mathrm{a}, \#}$ \\ ${ }^{a}$ Department of Ophthalmology, Affiliated Hospital of inner Mongolia University for the nationalities, Neimenggu 028050, China.
}

\begin{abstract}
Background: The pathological development of Diabetic retinopathy (DR) is an intricate process with multiple steps, and implicates a battery of dysregulated genes and regulators. We aimed to investigate the pathological mechanism of DR involving the regulatory association among H19, miR-29b and FOX04.

Methods: Sprague-Dawley (SD) rats were used to establish the diabetes mellitus (DM) model by injection streptozotocin. The levels of miR-29b, H19 and the mRNA level of FOXO4 were detected by qRT-PCR. The expression of FOXO4 at protein level was assessed by Western blot assay. The apoptosis of rMC-1 cells were analyzed by flow cytometry. To further investigate the relationship between H19 and miR-29b, the RIP and pull down assays were performed.

Results: The expression of H19 and FOXO4 were enhanced obviously, and the level of miR-29b was decreased in the retina tissues of DM rats and high glucose (HG)-treated rMC-1 cells. HG, as well as over-expression of H19, stimulated the apoptosis of rat retinal Müller cells. Knockdown of H19 reversed HG stimulation on cell apoptosis and FOXO4 up-regulation. RIP assay and RNA pull-down assay indicated that H19 was a target of miR-29b and inhibition of miR-29b reversed H19 down-regulation effect on cell apoptosis and FOXO4 expression. Down-regulation of FOXO4 reversed the effect of miR-29b knockdown on cell apoptosis.

Conclusion: MiR-29b targeting lncRNA-H19 mediates the apoptosis of rat retinal Müller cells via regulating FOX04 in diabetic retinopathy.
\end{abstract}

Keywords: LncRNA-H19; miR-29b; FOX04; Retinal Müller cell apoptosis; Diabetic retinopathy

\section{INTRODUCTION}

As the one of the most frequent metabolic disorder caused by multiple factors, diabetes mellitus (DM) is a disease with high incidences worldwide and the increased prevalence in the last decade ${ }^{[1,2]}$. With the hallmark of chronic hyperglycemia attributing to the defects in insulin secretion, action or both, DM will implicate the function of multi-organs, such as kidney, blood vessels, eyes and heart ${ }^{[3,4]}$, which causes great pain for the DM patients and their family due to the complications of DM. Diabetic retinopathy (DR) is one of the most vital DM complications with harrying approximately $20 \%$ of adult DM patients, which is one of the etiological factor of vision loss worldwide ${ }^{[5]}$. The development of DR presents with highly complex

\footnotetext{
${ }^{\#}$ Contributed equally.

*Corresponding author: Qiu-li Zhang

Mailing address: Department of Ophthalmology, Affiliated Hospital of inner Mongolia University for the nationalities, No 1742, HuoLinHe Street, Tongliao, Neimenggu 028050, China.

E-mail: Qiu_lizhang@126.com

Tel: +86-0475-8314342

Received: 7 November 2018 Accepted: 10 December 2018
}

pathogenesis attributing to implicating multiple interconnected mechanisms resulting in cellular damage, even and apoptosis in the retina ${ }^{[6]}$. Hence, it is essential to explore the potential mechanism of DR to bring the novel insight for the clinical therapy.

As a class of non-coding RNA without the capacity of coding protein, long non-coding RNA (lncRNA) is longer than $200 \mathrm{nts}{ }^{[7,8]}$. Emerging researches indicated that IncRNA were involved in genetic, epigenetic and post-transcriptional modulation in various processes or mechanisms of biology and pathology ${ }^{[9-12]}$. LncRNA-H19 located at 11p15.5 locus is expressed highly in fetus but decreasingly after birth, and also is implicated in the mechanisms of diverse diseases ${ }^{[13]}$. For instance, H19 serves as a carcinogenic gene in gastric cancer ${ }^{[14]}$, colorectal cancer ${ }^{[15]}$ and glioma cells ${ }^{[16]}$. In addition, Zhao reported that H19 promotes the apoptosis of hippocampal neuron in streptozotocin-induced DM rat model via Wnt signaling ${ }^{[17]}$. However, the underlying mechanism of H19 involved in DR remains unclear.

As another class of non-coding RNA without the capacity of coding protein, microRNA (miRNA) is always function in post-transcriptional processes via binding with 
3'UTR of target mRNAs to inhibit mRNA translation or degrade mRNA ${ }^{[18]}$, and is well-documented regulator in various diseases ${ }^{[19-21]}$. In the recent studies, lager numbers of miRNAs have been reported to mediate the occurrence and development of DM-related disorders, including miR-29b which has been reported to be expressed aberrantly in DM ${ }^{[2]}$. Moreover, miR-29b was predicted to have the binding site with H19. Hence, we hypothesize that miR-29b participates in the underlying mechanism of H19 in DR.

Forkhead box 04 (FOX04) has reported to be closely associated with the cell apoptosis in several diseases ${ }^{[23,24]}$. In experimental retinal detachment, the expression of FOXO4 was increased by Resveratrol treatment to prevent vision loss ${ }^{[25]}$. Thus, we aimed to investigate the pathological mechanism of DR involving the regulatory association among H19, miR-29b and FOXO4.

\section{METHODS}

\section{The establishment of DM rat model}

Sprague-Dawley (SD) rats (age: 4-6 weeks, sex: male) purchased from Shanghai Bioray Laboratories were randomly divided into two groups: the control group $(n=6)$ and the DM group $(n=6)$. All rats were raised in the uniform conditions with adequate water and food. After acclimatization for one week, the rats in DM group were intraperitoneally injected with streptozotocin (STZ, $60 \mathrm{mg} / \mathrm{kg}$ in $0.1 \mathrm{~mol} / \mathrm{l}$ citrate buffer) to induce DM and the rats in the control group were injected with $0.1 \mathrm{~mol} / \mathrm{l}$ citrate buffer. The concentration of blood glucose detected $72 \mathrm{~h}$ after the injection more than $16.7 \mathrm{mM}$ was regarded as that DM model was established successfully. Rat retinal tissues were collected immediately following rats were killed after $72 \mathrm{~h}$ of injection. All experiments in this study obtained the permission of the Animal Care and Use Committee of the Affiliated Hospital of inner Mongolia University for the Nationalities.

\section{Cell culture and treatment}

Rat retinal Müller cells (rMC-1) were obtained from EK-Bioscience (Biotechnology Co., Ltd. Shanghai Enzyme Research) and seeded in the 24-well plates and cultured in RPMI 1640 medium containing 10\% FBS with $5 \% \mathrm{CO} 2$ at $37^{\circ} \mathrm{C}$. To induce the DM Müller cell model, normal glucose $(5.5 \mathrm{mM})$ or high glucose (HG, $25 \mathrm{mM}$ ) was used to stimulate rMC-1 cells for $24,48,72$ and $96 \mathrm{~h}$. In the following experiment, rMC-1 cells were seeded in a 96-well plate and maintained for $24 \mathrm{~h}$. After pcDNA-H19/pcDNA was transfected into the rMC-1 cells with Lipofectamine 2000 reagent (Invitrogen), the cells were treated with normal glucose $(5.5 \mathrm{mM})$ for 72 h.

In the following experiment, $\mathrm{rMC}-1$ cells were seed in a 96-well plate and maintained for $24 \mathrm{~h}$. After si-ctrl/siH19 (si-control/si-H19), miR-29b inhibitor/NC and/or si-FOX04 were transfected into the rMC-1 cells with Lipofectamine 2000 reagent (Invitrogen), the cells were treated with HG for $72 \mathrm{~h}$. All transfected genes were synthesized by Shanghai Yingjun Co., Ltd. (China).

\section{Assessment of the expression of miR-29b, H19 and FOXO4}

The levels of miR-29b, H19 and the mRNA level of FOXO4 were detected by qRT-PCR. Total RNAs of retinal tissues and rMC-1 cells were isolated by TRIzol reagent (Invitrogen) following its manufacturer's instructions. Complementary DNA (cDNA) was synthesized by the reverse transcription of RNA with commercially available kit (iScript ${ }^{\mathrm{TM}}$ ). qRT-PCR was performed using SYBR ${ }^{\circledR}$ Premix DimerEraser kit (TaKaRa) on the instrument ABI 7000 PCR (Applied Biosystems, Japan). GAPDH acted as the control gene for H19 and FOX04,
A

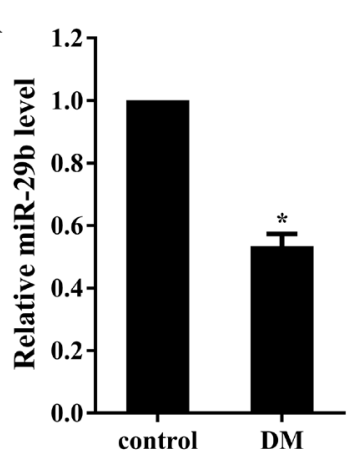

B

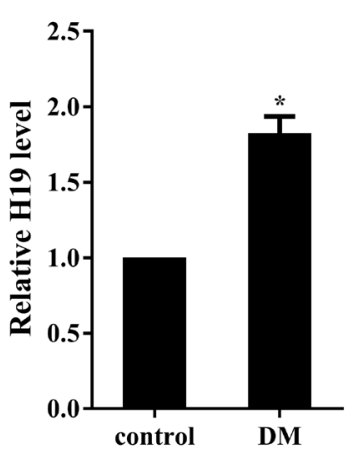

C

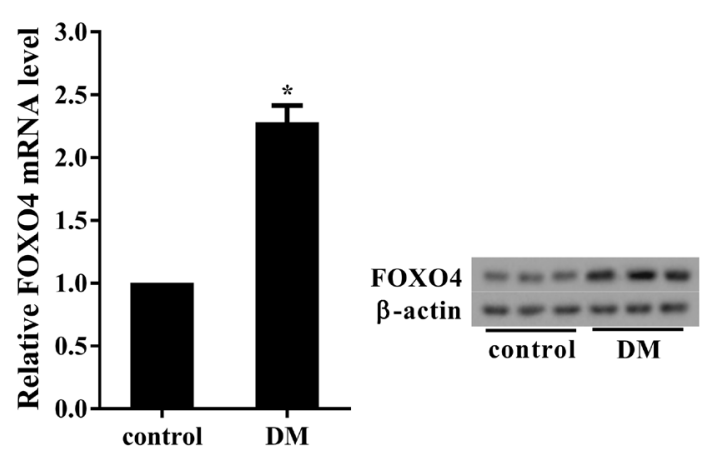

Figure 1. The expression profile of RNAs in retina tissues of DM rat. (A) The expression of miR-29b in retina tissues was investigated by qRT-PCR. (B) The expression of IncRNA-H19 in retina tissues was determined using qRT-PCR. (C) The expression of FOXO4 in retina tissues was detected at both the mRNA and protein levels. ${ }^{*} \mathrm{P}<0.01$ vs. control. 
A

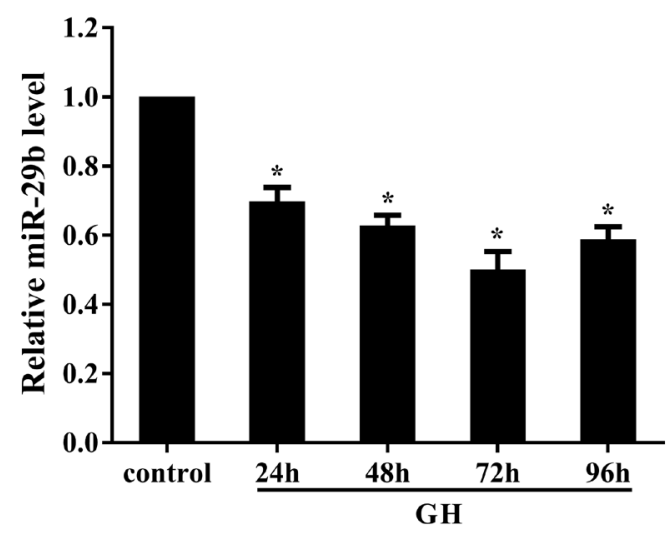

C

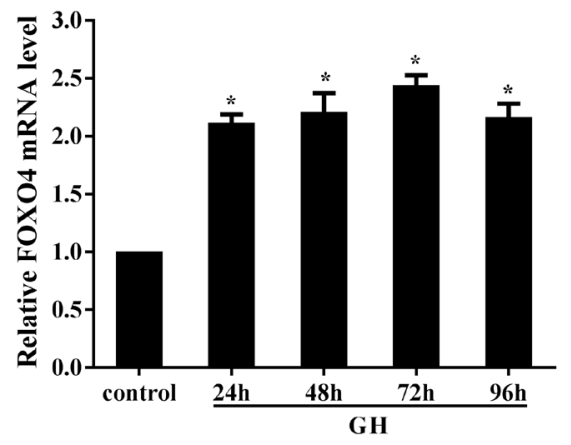

B

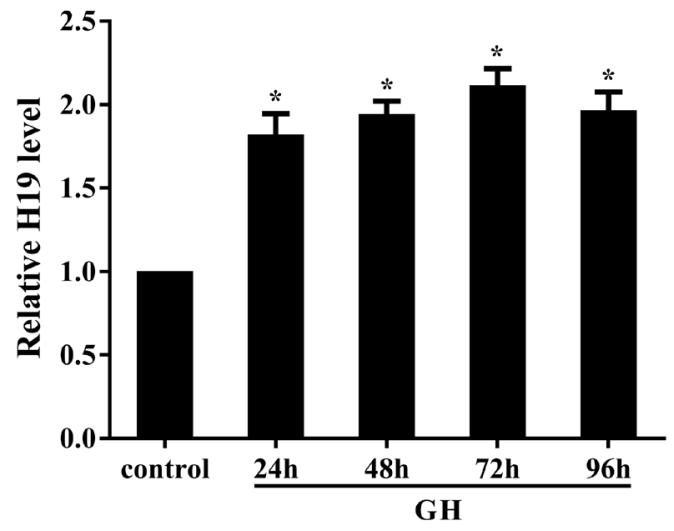

FOXO4

$\beta$-actin

Ctrl 24h 48h 72h 96h

GH

Figure 2. High glucose induced the dysregulation of miR-29b, H19 and FOXO4 and apoptosis in rat retinal Müller cells. (A) The expression of miR-29b in Müller cells was determined using qRT-PCR. (B) The expression of H19 in Müller cells was determined using qRT-PCR. (C) The expression of FOXO4 in rat Müller cells was detected at both the mRNA and protein levels. (D) The apoptosis of rMC1 cells was assessed using FCM assay. ${ }^{*} \mathrm{P}<0.01$ vs. control.

$\beta$-actin was used as the control gene for miR-29b. $2^{-\Delta \Delta C T}$ method was performed to qualify the relative amount of mRNAs.

The expression of FOXO4 at protein level was assessed by Western blot assay. The proteins extracted by RIPA lysis buffer and centrifugation were separated with an SDS/PAGE (12\% gel) and then transferred into the membrane which then was incubated with antibodies against FOXO4 (Sigma) and $\beta$-actin as internal control at $4^{\circ} \mathrm{C}$ for $24 \mathrm{~h}$, followed by maintained with the secondary antibodies for another $2 \mathrm{~h}$. SuperSignal West Pico chemiluminescence kit (Thermo Scientific) was used to visualize the protein bands.

\section{The apoptosis of cell}

The apoptosis of $\mathrm{rMC}-1$ cells were determined by FITC-Annexin V Apoptosis Detection Kit (Biosciences, USA) in accordance with previous report ${ }^{[26]}$. Briefly, rMC-1 cells with different treatments were cultured with $5 \% \mathrm{CO} 2$ at $37^{\circ} \mathrm{C}$ for $48 \mathrm{~h}$ in a 96 -well plate. The cells were collected and stained by PI (Sigma) for 30 min, followed by that the level of apoptosis was detected by FITC-Annexin V Apoptosis Detection Kit ground on the double staining with FITC-Annexin V and PI. The apoptotic rMC-1 cell was analyzed with a flow cytometry (FCM; FACScan).

\section{RNA immunoprecipitation (RIP) assay}

RNA-induced silencing complex (RISC) with an essential component that is Ago2 plays an important role in miRNAs putting to use their functions for gene silencing ${ }^{[27,28]}$. We hypothesized that $\mathrm{H} 19$ and miR-29b might be in the same RNA-induced silencing complex. RIP assay was performed following previous report ${ }^{[29]}$ by Magna RIP RNA-Binding Protein Immunoprecipitation Kit (Millipore, Billerica, MA, USA). Briefly, rMC-1 cells were lysed and then maintained with RIP buffer supplemented with magnetic beads conjugated with human anti-Ago 2 antibody (Abcam, Cambridge, MA, USA) and normal mouse IgG (negative control; Millipore). Ago2 in RNA-binding complex or total RNA (input control) was detected using IP-western. The H19 and miR- 
A

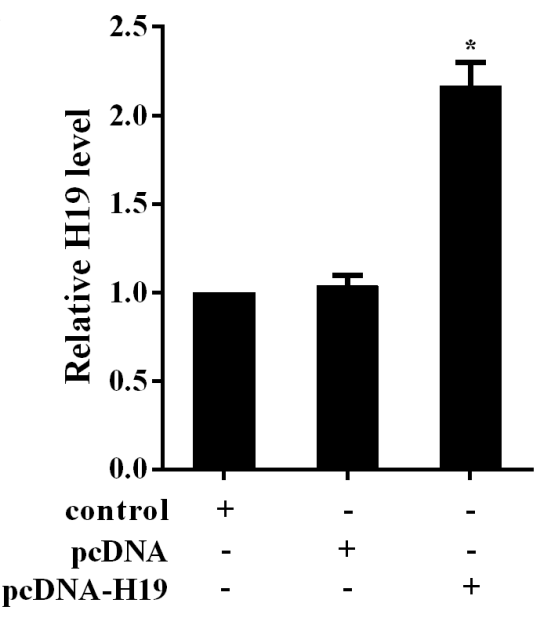

B

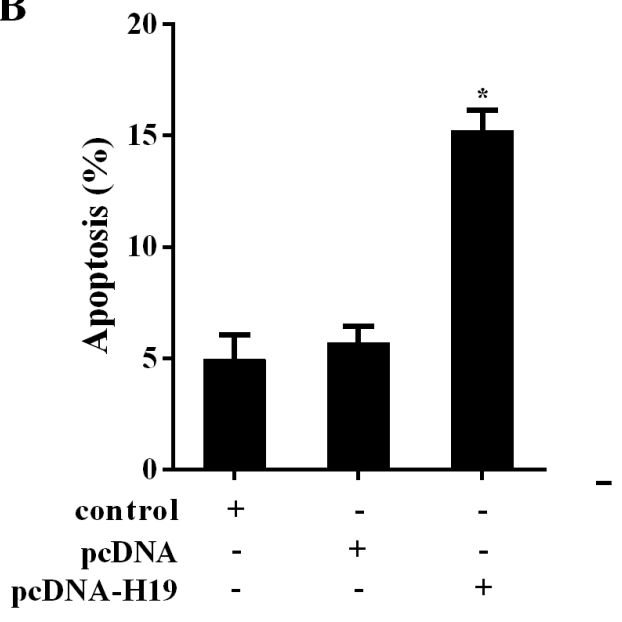

Figure 3. Up-regulation of H19 promoted the apoptosis of rMC-1 cells. (A) H19 was over-expressed in rMC-1 cells. (B) The apoptosis of Müller cells were assessed by FCM assay. ${ }^{*} \mathrm{P}<0.01$ vs. pcDNA-H19.

29b of co-precipitated RNAs were examined by qRTPCR following the antibody were recovered by protein A/G beads.

\section{RNA pull-down}

To explore physical relation between H19 and Ago2, RNA pull-down assay were performed via H19 labeled with biotin as a probe to assess Ago2 from the complex via western blot and miR-29b by qRT-PCR. As the positive control, lncRNA-loc285194 (LOC) ${ }^{[30]}$ was also cloned and then the resultant plasmid DNA was linearized by restriction enzyme NotI. Biotin-labeled RNAs were reversely transcribed by using Biotin RNA Labeling Mix (Roche Diagnostics) and T7 RNA polymerase (Roche, Basel, Switzerland), followed by treated with RNase-free DNase I (Roche, USA) and purified with the RNeasy Mini Kit (Qiagen, Valencia, CA, USA) and were further used for co-precipitating RNA. Ago2 was assessed by western blot. The level of miR-29b in co-precipitated RNAs was determined using qRT-PCR [31].

\section{Statistical analysis}

All data were harvested and statistically analyzed by Graph Pad Prism software (Graph Pad Software, La Jolla, USA) and show as mean \pm standard deviation (SD) with at least three repeats. Student's t test was used to assess the difference of two groups, and one-way ANOVA was used to assess the difference of multiple groups. $\mathrm{P}<0.01$ was considered as statistically significant.

\section{RESULTS}

The levels of miR-29b, H19 and F0X04 were expressed aberrantly in diabetic retinopathy (DR) tissues.

SD rats were intraperitoneally injected with streptozotocin to establish the rat DM model. After the successful DM model, the retina tissues were harvested to determine the expressions of miR-29b, H19 and FOXO4. Compared with the control rats, the level of miR-29b was markedly decreased (Figure 1A), the expression level of H19 was significantly enhanced (Figure 1B), and the expression of FOXO4 was obviously elevated at mRNA and protein levels (Figure 1C) in DM rats.

High glucose induced the dysregulation of miR-29b, H19 and FOXO4 and apoptosis in rat retinal Müller cells.

The Müller cells of rat were treated with high glucose (25 mM) for different times ( $24 \mathrm{~h}, 48 \mathrm{~h}, 72 \mathrm{~h}$ and 96 h). The expression of miR-29b was gradually reduced as time goes by, and reached a lowest level at $72 \mathrm{~h}$ in HG-treated Müller cells (Figure 2A). On the contrary, the expressions of $\mathrm{H} 19$ and FOXO4 were gradually increased over time, and reached their maximum levels at $72 \mathrm{~h}$ (Figure 2B\&C). Moreover, the apoptosis of Müller cells was gradually elevated with the time prolongation of HG treatment (Figure 2D).

\section{Over-expression of $\mathrm{H} 19$ promoted the apoptosis of rMC-1 cells.}

The rMC-1 cells were transfected with pcDNA-H19 and cultured with $5.5 \mathrm{mM}$ glucose. The expression of H19 in rMC-1 cells transfected by pcDNA-H19 in was remarkably higher than that in $\mathrm{rMC}-1$ cells without 
the transfection of pcDNA-H19 (Figure 3A). The apoptosis of rat Müller cells was greatly enhanced with the over-expression of H19 in rMC-1 cells (Figure 3B).

\section{MiR-26b could target bind with H19.}

In accordance with bioinformatics database, miR-29b was predicted to have a binding site with $\mathrm{H} 19$ (Figure $4 \mathrm{~A}$ ). To further investigate the relationship between H19 and miR-29b, the RIP and pull down assays were performed. The result of RIP assay indicated that Ago2 protein in the $\mathrm{rMC}-1$ cell lysates was precipitated by anti-Ago2 in IP-western, and the levels of H19 and miR29b in Ago2 precipitation were significantly higher than that in the control (Figure 4B). As shown in Figure 4C, Ago 2 were examined in H19 pulled down complex using western blot assays, and miR-29b was found to be enriched more significantly in the same pellet than that in LOC pull-down complex, revealing that H19 is a positive miR-29b-targeting IncRNA.

H19 mediated HG-induced apoptosis of Müller cells via miR-29b regulating FOX04.

As shown in Figure 5\&6, HG could obviously enhance the expression of FOXO4 at both mRNA and protein levels in rMC-1 cells, as well as the apoptosis of Müller cells. After rMC-1 cells were transfected by si-H19 and then treated with $\mathrm{HG}, \mathrm{HG}$-induced effects on the enhancement of FOXO4 expression and cell apoptosis were reversed by silence of H19. Furthermore, rMC-1 cells were transfected by si-H19 and miR-29b inhibitor and then treated with HG could abrogate the effect of H19 knockdown on FOXO4 expression and Müller cell apoptosis. Meanwhile, down-regulation of FOXO4 in rMC- 1 cells transfected by si-H19 and miR-29b inhibitor also could alleviate the effect of miR-29b knockdown on the apoptosis of Müller cell.

\section{DISCUSSION}

Diabetic retinopathy is the injury of vessel and neurology in the retina resulted from metabolic disorders in $\mathrm{DM}^{[25]}$. The pathological development of DR is an intricate process with multiple steps, and implicates a battery of dysregulated genes and regulators including lncRNAs and miRNAs. Similarly in our study, the expression of H19, miR-29b and FOXO4 were dysregulated in the retina tissues of DM rats. It presents clear that HG levels caused by metabolic disorders of diabe-

\section{A miR-29b 3, UUGUG---ACUAAAGUUUACCACGAU 5} H19 GAACCCACAACAUGAAAG-A I

B
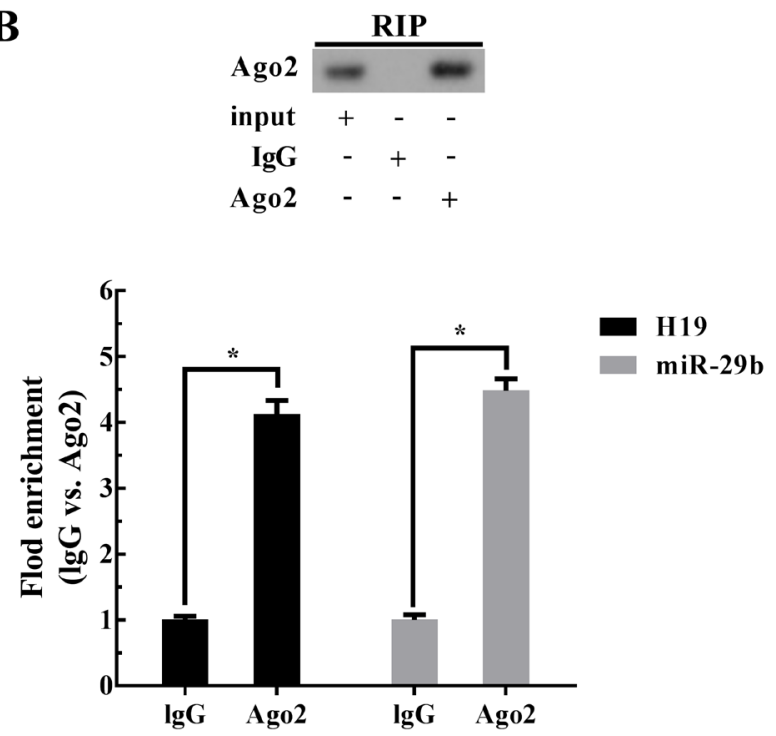

C
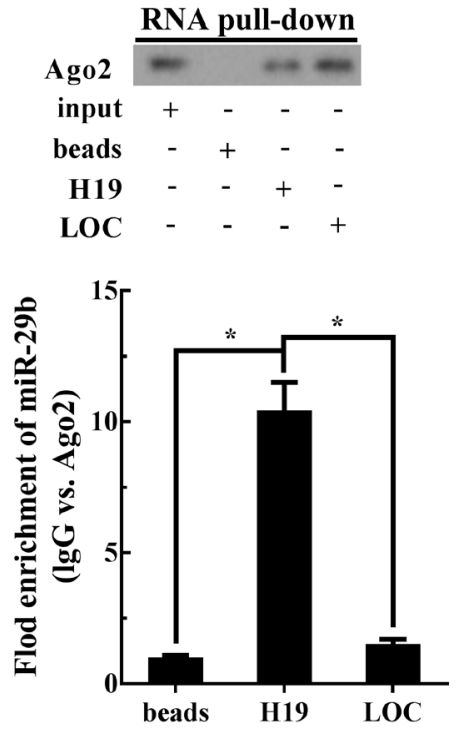

Figure 4. H19 could target to with miR-29b. (A) Predicted binding sites between H19 and miR-29b. (B) Cellular lysates of Müller cells were used for RIP assay with Ago2 antibody. The level of Ago2 was detected using IP-western (up panel). H19 and miR-29b levels were detected using qRT-PCR (down panel). ${ }^{*} \mathrm{P}<0.01$ vs. IgG. (C) Verification of H19 and miR-29b in the same RISC complex by RNA pull down assay. Pull-down of Ago2 was determined by western blot assay (up panel). MiR-29b in the RNA pulled down samples was detected using qRT-PCR (down panel). ${ }^{*} \mathrm{P}<0.01$ vs. beads; ${ }^{*} \mathrm{P}<0.01$ vs. LOC. 


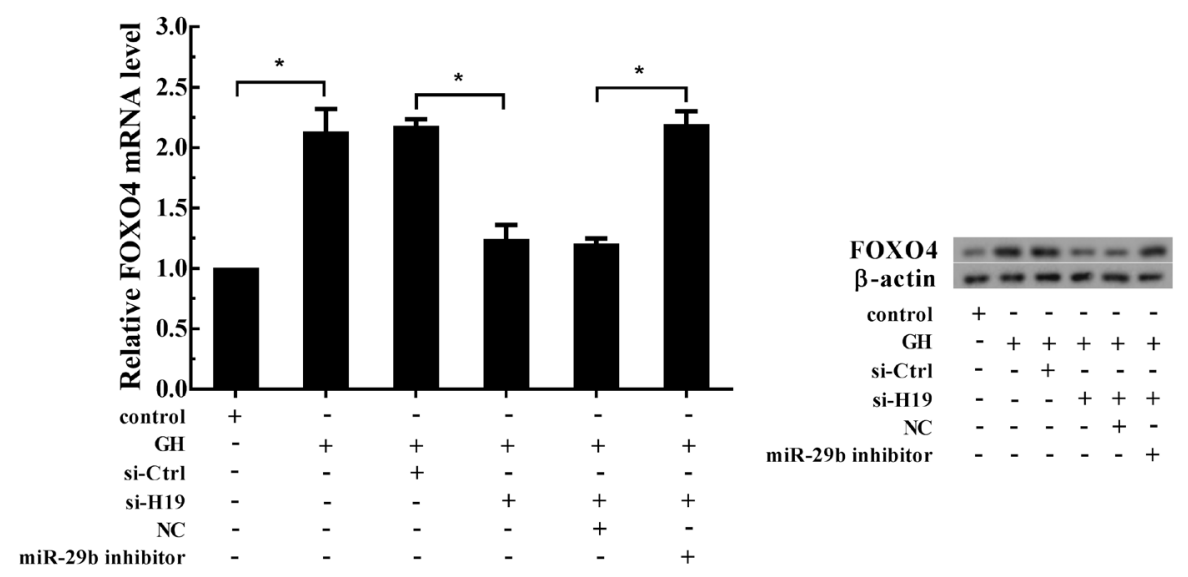

Figure 5. H19 regulated FOXO4 expression via miR-29b. Müller cell lines were transfected with si-Ctrl/si-H19 or si-H19+NC/siH19+miR-29b inhibitor, followed by treated by HG. The expression of FOXO4 in rMC-1 cells was detected both at the mRNA and protein levels. ${ }^{*} \mathrm{P}<0.01$ vs. control; ${ }^{*} \mathrm{P}<0.01$ vs. si-Ctrl; ${ }^{*} \mathrm{P}<0.01$ vs. si-H19+NC.

tes are the direct cause of neural cell injury in retinal tissues ${ }^{[32]}$. In our study, HG stimulated the apoptosis of rat retinal Müller cells, as well as induced the aberrant expression of expression of H19, miR-29b and FOXO4. Hence, we inferred that the levels of H19, miR-29b and FOXO4 might be correlated with the apoptosis of rat retinal Müller cells.

To further investigate the mechanism of DR involving these genes, we concluded from recent reports that

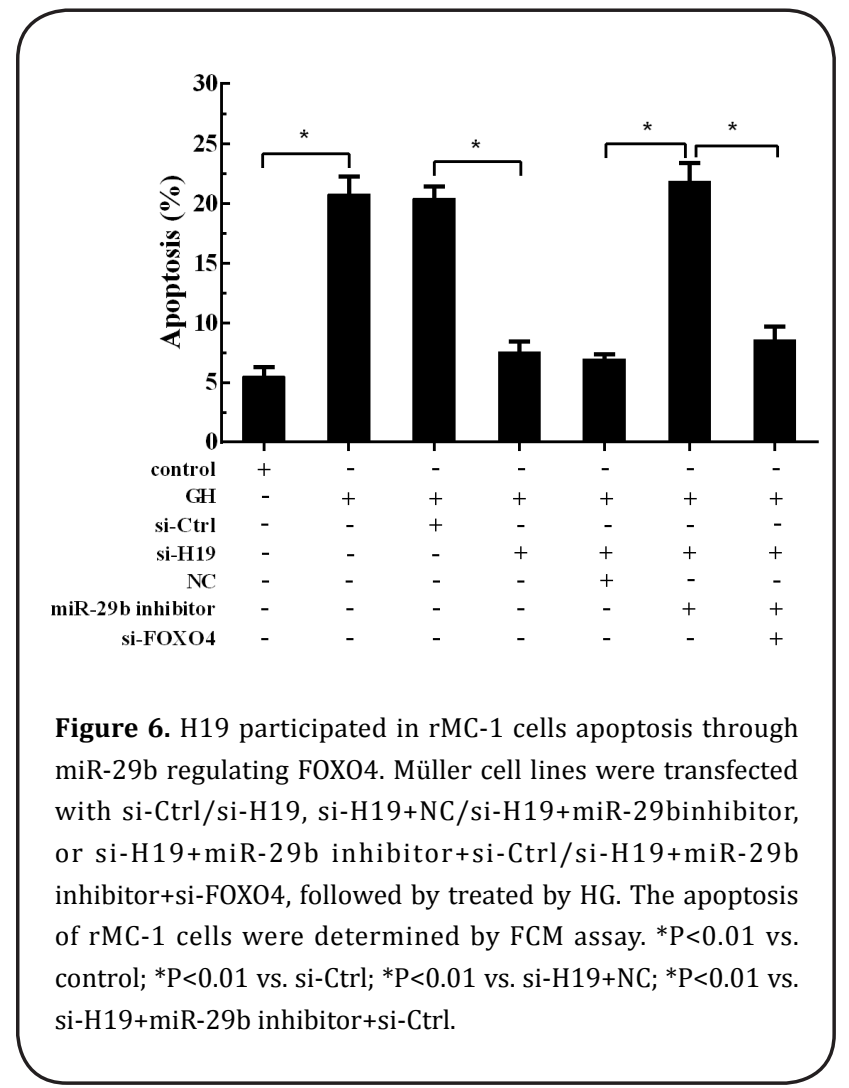

H19 plays important roles in the apoptosis of various cells, such as cardiomyocytes ${ }^{[33]}$, gastric cancer cells ${ }^{[34]}$, osteoblastic cell ${ }^{[35]}$, and hippocampal neuron of DM rat $^{[17]}$. In our study, we also found that over-expression of H19 induced the apoptosis of rat retinal Müller cells and knockdown of $\mathrm{H} 19$ reversed HG stimulation on cell apoptosis and FOXO4 up-regulation, which means that HG stimulated the apoptosis of rat retinal Müller cells with $\mathrm{H} 19$ up-regulation. On the other hand, miR-29b is another dysregulated regulator involved in diabetes. In DM rats, the level of miR-29b was increased by diabetes, but acute contractile stimulus reduced miR-29b level in muscle from diabetic rats ${ }^{[36]}$. Moreover, miR$29 \mathrm{~b}$ presented the protective effect on dorsal root ganglia neurons of diabetic rat ${ }^{[22]}$. In our precious study, miR-29b acted as a biomarker was regulated by MIAT and further modulated rat retinal Müller cell apoptosis in $\mathrm{DR}^{[37]}$. In present study, RIP assay and RNA pulldown assay indicated that $\mathrm{H} 19$ was a target of miR-29b and inhibition of miR-29b reversed H19 down-regulation effect on cell apoptosis and FOXO4 expression, which indicated that $\mathrm{H} 19$ mediated rat retinal Müller cell apoptosis and FOXO4 expression via target regulating miR-29b.

FOXO4 is a number of forkhead box proteins of 0 class proteins (FOXOs) family that were known to act as the transcription factors to drive multifarious physiological processes in cells, including proliferation, DNA damage, differentiation and apoptosis ${ }^{[38,39]}$. In addition, FOXO4 was reported to be the major pathogenic factor to mediate endothelial damage under hyperglycemia ${ }^{[0,41]}$. Our study revealed that down-regulation of FOXO4 suppressed cell apoptosis in rMC-1 cells, which was consistent with others reports ${ }^{[42]}$. Moreover, down-reg- 
ulation of FOXO4 reversed the effect of miR-29b knockdown on cell apoptosis, indicating that the relationship between miR-29b and rat retinal Müller cell apoptosis depended on FOXO4.

In summary, miR-29b targeting lncRNA-H19 mediates the apoptosis of rat retinal Müller cells via regulating FOXO4 in diabetic retinopathy.

\section{Competing interests}

The authors declare that there are no competing interests associated with the manuscript.

\section{Acknowledgment}

This study was approved by National Natural Science Foundation of China (no. 81660734) and Natural Science Foundation Committee of Inner Mongolia (2018MS08118).

\section{REFERENCES}

1. Amp, T. L. D. Diabetes: a 21st century challenge.

2. Whiting, D. R., Guariguata, L., Weil, C., and Shaw, J. (2011) IDF diabetes atlas: global estimates of the prevalence of diabetes for 2011 and 2030. Diabetes Res Clin Pract 94, 311-321

3. Association, A. D. (2013) American Diabetes Association Diagnosis and classification of diabetes mellitus.

4. Merovci, A., Solis-Herrera, C., Daniele, G., Eldor, R., Fiorentino, T. V., Tripathy, D., Xiong, J., Perez, Z., Norton, L., Abdul-Ghani, M. A., and DeFronzo, R. A. (2014) Dapagliflozin improves muscle insulin sensitivity but enhances endogenous glucose production. J Clin Invest 124, 509514

5. Bourne, R. R., Jonas, J. B., Flaxman, S. R., Keeffe, J., Leasher, J., Naidoo, K., Parodi, M. B., Pesudovs, K., Price, H., White, R. A., Wong, T. Y., Resnikoff, S., Taylor, H. R., and Vision Loss Expert Group of the Global Burden of Disease S. (2014) Prevalence and causes of vision loss in high-income countries and in Eastern and Central Europe: 1990-2010. Br J Ophthalmol 98, 629-638

6. Robinson, R., Barathi, V. A., Chaurasia, S. S., Wong, T. Y., and Kern, T. S. (2012) Update on animal models of diabetic retinopathy: from molecular approaches to mice and higher mammals. Dis Model Mech 5, 444-456

7. Batista, P. J., and Chang, H. Y. (2013) Long noncoding RNAs: cellular address codes in development and disease. Cell 152, 1298-1307

8. Ling, H., Fabbri, M., and Calin, G. A. (2013) MicroRNAs and other non-coding RNAs as targets for anticancer drug development. Nat Rev Drug Discov 12, 847-865

9. Tripathi, V., Shen, Z., Chakraborty, A., Giri, S., Freier, S. M., Wu, X., Zhang, Y., Gorospe, M., Prasanth, S. G., Lal, A., and Prasanth, K. V. (2013) Long noncoding RNA MALAT1 controls cell cycle progression by regulating the expres- sion of oncogenic transcription factor B-MYB. PLoS Genet 9, e1003368

10. Yu, W., Gius, D., Onyango, P., Muldoon-Jacobs, K., Karp, J., Feinberg, A. P., and Cui, H. (2008) Epigenetic silencing of tumour suppressor gene $\mathrm{p} 15$ by its antisense RNA. Nature 451, 202-206

11. Qi, P., and Du, X. (2013) The long non-coding RNAs, a new cancer diagnostic and therapeutic gold mine. Mod Pathol 26, 155-165

12. Yan, B., Yao, J., Liu, J. Y., Li, X. M., Wang, X. Q., Li, Y. J., Tao, Z. F., Song, Y. C., Chen, Q., and Jiang, Q. (2015) IncRNA-MIAT regulates microvascular dysfunction by functioning as a competing endogenous RNA. Circ Res 116, 1143-1156

13. Wang, S. H., Ma, F., Tang, Z. H., Wu, X. C., Cai, Q., Zhang, M. D., Weng, M. Z., Zhou, D., Wang, J. D., and Quan, Z. W. (2016) Long non-coding RNA H19 regulates FOXM1 expression by competitively binding endogenous miR-3423p in gallbladder cancer. J Exp Clin Cancer Res 35, 160

14. Liu, G., Xiang, T., Wu, Q. F., and Wang, W. X. (2016) Long Noncoding RNA H19-Derived miR-675 Enhances Proliferation and Invasion via RUNX1 in Gastric Cancer Cells. Oncol Res 23, 99-107

15. Tsang, W. P., Ng, E. K., Ng, S. S., Jin, H., Yu, J., Sung, J. J., and Kwok, T. T. (2010) Oncofetal H19-derived miR-675 regulates tumor suppressor RB in human colorectal cancer. Carcinogenesis 31, 350-358

16. Shi, Y., Wang, Y., Luan, W., Wang, P., Tao, T., Zhang, J., Qian, J., Liu, N., and You, Y. (2014) Long non-coding RNA H19 promotes glioma cell invasion by deriving miR-675. PLoS One 9, e86295

17. Zhao, Y. H., Ji, T. F., Luo, Q., and Yu, J. L. (2017) Long non-coding RNA H19 induces hippocampal neuronal apoptosis via Wnt signaling in a streptozotocin-induced rat model of diabetes mellitus. Oncotarget, , 8, 6482764839

18. Feng, D. D., Zhang, H., Zhang, P., Zheng, Y. S., Zhang, X. J., Han, B. W., Luo, X. Q., Xu, L., Zhou, H., Qu, L. H., and Chen, Y. Q. (2011) Down-regulated miR-331-5p and miR-27a are associated with chemotherapy resistance and relapse in leukaemia. J Cell Mol Med 15, 2164-2175

19. Hermeking, H. (2012) MicroRNAs in the p53 network: micromanagement of tumour suppression. Nat Rev Cancer $12,613-626$

20. Issler, O., and Chen, A. (2015) Determining the role of microRNAs in psychiatric disorders. Nat Rev Neurosci 16, 201-212

21. Moy, R. H., Cole, B. S., Yasunaga, A., Gold, B., Shankarling, G., Varble, A., Molleston, J. M., tenOever, B. R., Lynch, K. W., and Cherry, S. (2014) Stem-loop recognition by DDX17 facilitates miRNA processing and antiviral defense. Cell 158, 764-777

22. Zhang, X., Gong, X., Han, S., and Zhang, Y. (2014) MiR-29b protects dorsal root ganglia neurons from diabetic rat. Cell Biochem Biophys 70, 1105-1111 
23. Wang, W., Zhou, P. H., and Hu, W. (2016) Overexpression of FOXO4 induces apoptosis of clear-cell renal carcinoma cells through downregulation of Bim. Mol Med Rep 13, 2229-2234

24. Li, J., Hu, L., Tian, C., Lu, F., Wu, J., and Liu, L. (2015) microRNA-150 promotes cervical cancer cell growth and survival by targeting FOX04. BMC Mol Biol 16, 24

25. Huang, W., Li, G., Qiu, J., Gonzalez, P., and Challa, P. (2013) Protective effects of resveratrol in experimental retinal detachment. PLoS One 8, e75735

26. Chen, J., and Regan, R. F. (2004) Heme oxygenase-2 gene deletion increases astrocyte vulnerability to hemin. Biochem Biophys Res Commun 318, 88-94

27. Gregory, R. I., Chendrimada, T. P., Cooch, N., and Shiekhattar, R. (2005) Human RISC Couples MicroRNA Biogenesis and Posttranscriptional Gene Silencing. Cell 123, 631640

28. Karginov, F. V., Conaco, C., Xuan, Z., Schmidt, B. H., Parker, J. S., Mandel, G., and Hannon, G. J. (2007) A Biochemical Approach to Identifying MicroRNA Targets. Proc. Natl. Acad. Sci. U. S. A. 104, 19291

29. Lu, H., He, Y., Lin, L., Qi, Z., Ma, L., Li, L., and Su, Y. (2016) Long non-coding RNA MALAT1 modulates radiosensitivity of HR-HPV+ cervical cancer via sponging miR-145. Tumour Biol 37, 1683-1691

30. Liu, Q., Huang, J., Zhou, N., Zhang, Z., Zhang, A., Lu, Z., Wu, F., and Mo, Y. Y. (2013) LncRNA loc285194 is a p53-regulated tumor suppressor. Nucleic Acids Res 41, 49764987

31. Zhang, A., Zhou, N., Huang, J., Liu, Q., Fukuda, K., Ma, D., Lu, Z., Bai, C., Watabe, K., and Mo, Y. Y. (2013) The human long non-coding RNA-RoR is a $\mathrm{p} 53$ repressor in response to DNA damage. Cell Res 23, 340-350

32. Sun, X., and Wong, D. (2016) Long non-coding RNA-mediated regulation of glucose homeostasis and diabetes. Am J Cardiovasc Dis 6, 17-25

33. Zhang, Y., Zhang, M., Xu, W., Chen, J., and Zhou, X. (2017) The long non-coding RNA H19 promotes cardiomyocyte apoptosis in dilated cardiomyopathy. Oncotarget 8,
28588-28594

34. Liu, G., Xiang, T., Wu, Q. F., and Wang, W. X. (2016) Curcumin suppresses the proliferation of gastric cancer cells by downregulating H19. Oncol Lett 12, 5156-5162

35. He, P., Zhang, Z., Huang, G., Wang, H., Xu, D., Liao, W., and Kang, Y. (2016) miR-141 modulates osteoblastic cell proliferation by regulating the target gene of IncRNA H19 and IncRNA H19-derived miR-675. Am J Transl Res 8, 1780-1788

36. Gerlinger-Romero, F., Yonamine, C. Y., Junior, D. C., Esteves, J. V., and Machado, U. F. (2017) Dysregulation between TRIM63/FBX032 expression and soleus muscle wasting in diabetic rats: potential role of miR-1-3p, -29a/b-3p, and -133a/b-3p. Mol Cell Biochem 427, 187199

37. Zhang, J., Chen, M., Chen, J., Lin, S., Cai, D., Chen, C., and Chen, Z. (2017) Long non-coding RNA MIAT acts as a biomarker in diabetic retinopathy by absorbing miR-29b and regulating cell apoptosis. Biosci Rep 37

38. Eijkelenboom, A., and Burgering, B. M. (2013) FOXOs: signalling integrators for homeostasis maintenance. Nat Rev Mol Cell Biol 14, 83-97

39. Accili, D., and Arden, K. C. (2004) FoxOs at the crossroads of cellular metabolism, differentiation, and transformation. Cell 117, 421-426

40. Tsuchiya, K., Tanaka, J., Shuiqing, Y., Welch, C. L., DePinho, R. A., Tabas, I., Tall, A. R., Goldberg, I. J., and Accili, D. (2012) FoxOs integrate pleiotropic actions of insulin in vascular endothelium to protect mice from atherosclerosis. Cell Metab 15, 372-381

41. Chuang, P. Y., Yu, Q., Fang, W., Uribarri, J., and He, J. C. (2007) Advanced glycation endproducts induce podocyte apoptosis by activation of the FOXO4 transcription factor. Kidney Int 72, 965-976

42. Zhang, L., Dong, L., Liu, X., Jiang, Y., Zhang, L., Zhang, X., Li, X., and Zhang, Y. (2014) alpha-Melanocyte-stimulating hormone protects retinal vascular endothelial cells from oxidative stress and apoptosis in a rat model of diabetes. PLoS One 9, e93433 